\title{
AN EXTENDED BDI MODEL FOR HUMAN BEHAVIORS: DECISION-MAKING, LEARNING, INTERACTIONS, AND APPLICATIONS
}

\author{
Young-Jun Son \\ Sojung Kim \\ Hui Xi \\ Santosh Mungle \\ Systems and Industrial Engineering \\ The University of Arizona \\ Tucson, AZ 85721, USA
}

\begin{abstract}
Modeling human decision-making behaviors under a complex and uncertain environment is quite challenging. The goal of this tutorial is to discuss an extended Belief-Desire-Intention (BDI) framework that the authors' research group has been developing last decade to meet such a challenge, integrating models and techniques (e.g. Bayesian Belief Network, Decision Field Theory, Depth First Search) available in the fields of engineering, psychology, computational science, and statistics. First, major modules of the extended BDI framework are discussed, where those modules represent cognitive functions (i.e. perception, goal seeking, planning, decision-making, execution) of an individual. Then, two extensions are considered, where the first one involves dynamic evolution of underlying modules over time (i.e. learning and forgetting), and the second one involves human interactions (e.g. competition, collaboration, compromise, accommodation, avoidance). To illustrate the proposed approach, various applications are used, such as emergency evacuation during bomb attack, driver and pedestrian behaviors, and cyber social network.
\end{abstract}

\section{INTRODUCTION}

Humans are known to be able to learn how to maximize probability of success and to converge towards optimal or near optimal decisions when confronted with spatial complexity, temporal complexity, or challenges that require a high degree of creativity (NSF 2008). Understanding and modeling such human behaviors under a complex and uncertain environment are extremely challenging. To tackle such a challenging problem, researchers in various fields have been putting significant efforts. Among those efforts, some focus on a very specific cognitive function (e.g. memory) in a simplified and confined environment while others take a holistic approach to develop a comprehensive modeling architecture (e.g. ACT-R (Act-R 2013), COGNET (MIT CogNet 2013), EPIC (Kieras and Meyer 1997), Soar (Soar 2013)) for human cognitive functions. To enable enhanced understanding and modeling of complex human behaviors, both approaches (bottom-up vs. top-down) are important and need to be integrated.

The goal of this tutorial is to discuss an extended Belief-Desire-Intention (BDI) modeling framework (Zhao and Son 2008; Lee, Son, and Jin 2010) that the authors' research group has been developing last decade to mimic human decision behaviors. While the original BDI developed by Bratman (1987) is a software model that has been widely used to program optimal behaviors of intelligent machines (agents) in the artificial intelligence community (Wikipedia 2013), the authors' group has employed the conceptual aspect of the original BDI and further developed it to represent human cognitive functions (e.g. perception, goal seeking, planning, decision-making, execution, learning, forgetting, interactions, among others), integrating various models and techniques (e.g. Bayesian Belief Network (BBN), Decision Field Theory, 


\section{Son, Kim, Xi, and Mungle}

Depth First Search) available in the fields of engineering, psychology, computational science, and statistics.

The extended BDI modeling framework (Zhao and Son 2008; Lee, Son, and Jin 2010) has been used to present human behaviors under various applications, such as 1) emergency evacuation under bomb attack in the Washington mall area, 2) emergency evacuation from factory fire, 3) various driver behaviors (e.g. route choice, lane changing, following lead vehicle, dilemma zone), 4) shopping at a mall, and 5) social network interactions in a cyberspace. Section 3 provides more details on each of these applications.

\section{OVERVIEW OF EXTENDED BDI FRAMEWORK}

\subsection{Major Modules of Extended BDI Framework}
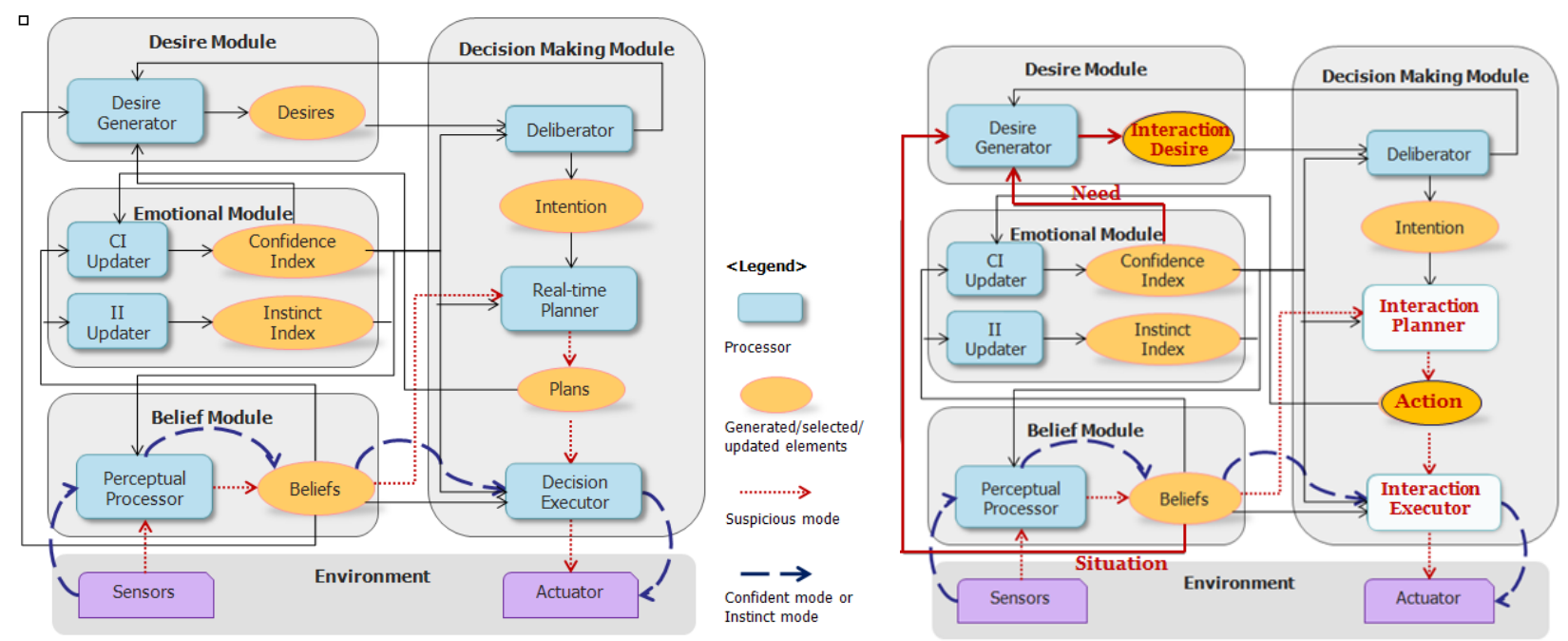

Figure 1: (a) Overview of extended BDI framework (Zhao and Son 2008; Lee, Son, and Jin 2010); (b) Adaptation of extended BDI framework to represent interactions between humans

Figure 1(a) depicts an overview of the extended BDI framework (Zhao and Son 2008; Lee, Son, and Jin 2010), whose major modules include 1) Belief Module, 2) Desire Module, 3) Decision Making Module, and 4) Emotion Module. The ellipse in each module (e.g. Beliefs in the Belief Module) stores elements that are generated, updated, or selected by the corresponding processor (e.g. Perceptual Processor in the Belief Module) represented by the square. Given dynamically changing, objective environmental conditions (depicted at the bottom of Figure 1(a)), the Perceptual Processor of an individual generates his subjective Beliefs. It is noted that given the same environmental condition, Beliefs of different individuals will be different depending on their cultural background as well as levels of experiences and knowledge. The Desire Module, based on the current Beliefs, then generates Desires. In our research, a Desire can be considered in two contexts. In the first context, it represents a long-term goal which a human being desires to achieve; in the second context, Desires contain multiple goals that an individual wants to achieve. The Deliberator in the Decision Making Module then generates an Intention (a short-term goal or one of multiple Desires) based on the Desire(s). The Real-time Planner in the Decision Making Module generates a plan (i.e. a series of actions to be taken needed to achieve the intention) based on his current Beliefs (on his capability and environmental conditions). After a plan is developed, the Decision Executor executes each action contained in the plan, which will affect the environment. If what is predicted during the planning stage is similar to what an individual faces during the execution stage, the Confidence Index (CI) in the Emotional Module increases, and he continues to execute actions until all the actions in the current plan are executed (i.e. confident mode). On the other hand, if there is a significant deviation be- 
tween what's predicted vs. the reality, the CI decreases. If the CI is below a threshold value, he develops a new plan (i.e. suspicious mode) based on the current environmental condition instead of completing the previously developed one. The Instinct Index (II) Updater increases the II in the event (e.g. decisionmaking under time pressure such as evacuation from factory fire) for which the above mentioned reasoning process is not possible; in this case, the human being follows his instinct (i.e. long-term memory), which is part of Beliefs.

\subsection{Theories and Techniques Used for Major Modules}

As the extended BDI framework that was discussed in Section 2.1 is conceptual, different techniques or tools can be employed to represent each of the modules (human cognitive functions). In the work of the authors' group (Lee, Son, and Jin 2010), BBN is used to present the Perceptual Processor. Figure 2(c) depicts an exemplary BBN for an individual under emergency evacuation from bomb attack, which represents how he perceives (believes) the risk and evacuation time for each of the considered paths (e.g. four paths at each intersection) considering their environmental conditions (i.e. intensity of fire, intensity of smoke, existence of policemen, existence of crowd, distance to the destination) (Lee, Son, and Jin 2010). To construct a BBN for different individuals, a behavior data needs to be collected under varying environmental conditions. In our previous works, we have collected behavior data from human-in-the-loop experiments conducted in a CAVE-based virtual reality environment (Lee, Son, and Jin 2010; Vasudevan and Son 2011) or a PC simulation (Son and Jin 2006), or via survey (Celik et al. 2011). Major advantages of BBN are 1) its ability to handle uncertainty, 2) its ability to handle both deductive reasoning (top-down reasoning) (which is used in Figure 3(c)) as well as inductive reasoning (bottom-up reasoning), and 3) its ability to handle situations with missing information.

To represent the Real-time Planner in our work, an extended version of Decision Field Theory (DFT) (Busemeyer and Townsend 1993) together with a probabilistic version of Depth First Search technique is used. The DFT is shown in (1), whose major elements are explained below:

- $\quad P(t)$ represents a preference state at time $t$ (for each of $m$ options considered in decision-making),

- $h$ is a time step,

- $S$ represents the effects of memory as well as the interactions among different options,

- $\quad M(t)(m \times n$ matrix for $m$ options and $n$ attributes) represents the subjective perceptions of an individual. In our work, values of $M$ matrix are inferred from the BBN (constructed for each individual) (see Figure 3(c)),

- $W(t)$ allocates the weights of attention corresponding to each of $n$ attribute considered at time $t$, and it changes over time according to a stationary stochastic process (a Bernoulli distribution has been used in our works).

$$
P(t+h)=S P(t)+C M(t+h) W(t+h)
$$

It is noted that elements of $M(t)$ and $W(t)$ are stochastic, so the DFT equation needs to be replicated multiple times. Therefore, the output from the DFT is a choice probability for each of the considered $n$ options. Figure 2 depicts how BBN and DFT are used together with depth first search to represent the Real-time Planner. Figures 2(a) and 2(b) depict a satellite image of the Washington DC mall area and its corresponding graph representation, respectively. It is supposed that an individual located in node $e$ (current location) intends to find a best path to node $o$ (destination) considering his risk handling behavior (represented by his corresponding BBN shown in Figure 2(c)). At each intersection starting from node e, BBN is first used to infer risk and evacuation time for each path using the BBN, whose output is then used by DFT to obtain choice probability for each path. Then, random sampling technique is used to select a path (in Figure 2, it is assumed that a path from node $e$ to node $j$ is selected). From node $j$, the same process (BBN, DFT, and random sampling) is repeated, where the only difference is that only limited environ- 
mental conditions (e.g. distance to the destination) are considered as the human is physically still in node $e$ and does not have access to all the environmental conditions (e.g. intensity of fire and smoke, existence of policemen and crowd) of paths from node $j$. Figure 2(d) depicts the planned evacuation paths (in lighter color) from node $e$ to node $o$.
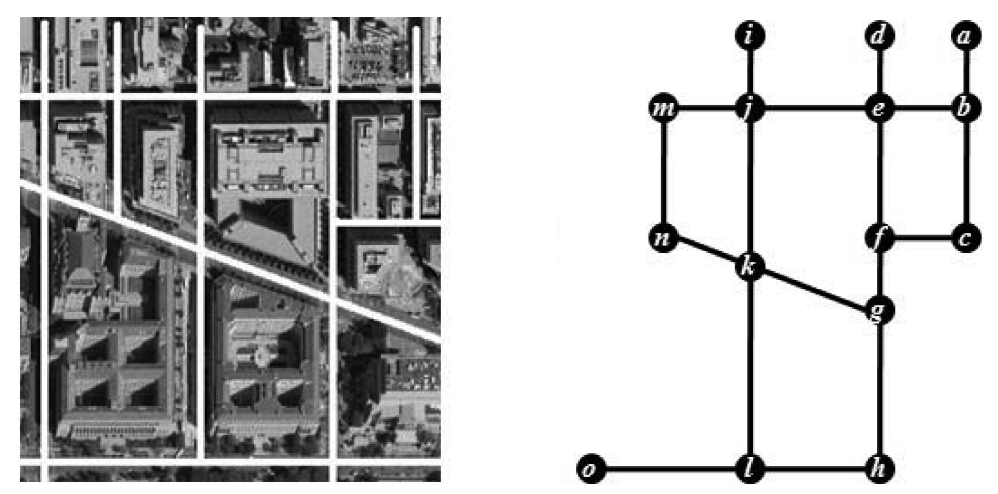

口
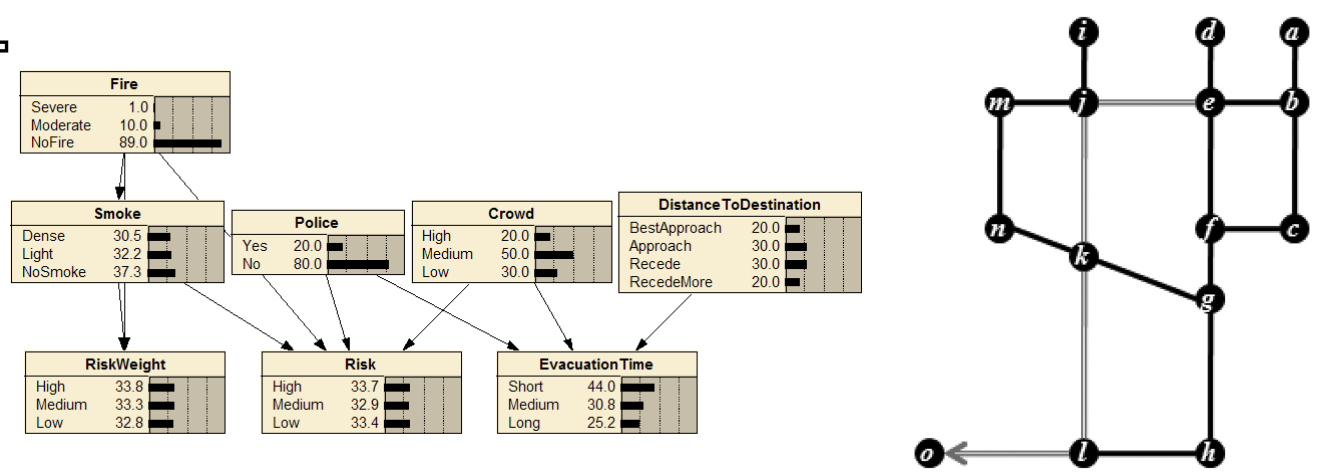

Figure 2: Illustration of multi-stage real-time planning under an emergency evacuation scenario: (a) Satellite image of Washington DC mall area, (b) graph representation of the area, (c) BBN to present perception of an individual, and (d) planned evacuation paths from node $e$ (current location) to node $o$ (destination) (Lee, Son, and Jin 2010)

Finally, empirical functions are developed to represent both the CI Updater and the II Updater. (2) depicts the CI at time $t$, where $\alpha$ represents the effect of the previous CI and $d_{t}^{y}$ represents the deviation between what was predicted about the environment of option $y$ during the planning stage and its actual environment experienced during the execution stage (Lee, Son, and Jin 2010). As shown in (3), the deviation is calculated for all $A$ number of attributes, where $m_{a}^{y}(t)$ is the perceived value of attribute $a$.

$$
\begin{gathered}
C I_{t}=\alpha e^{-d_{t}^{y}}+\left(\begin{array}{ll}
1 & \alpha
\end{array}\right) \in I_{t-1} \\
d_{t}^{y}=\sum_{a=1}^{A}\left|\left(m_{a}^{y}(t)-m_{a}^{y}(t-1)\right)\right|
\end{gathered}
$$




\section{Son, Kim, Xi, and Mungle}

\subsection{Learning and Forgetting under the Extended BDI Framework}

While Section 2.1 discussed underlying modules of the extended BDI framework to mimic major human cognitive functions (e.g. perception, goal seeking, planning, decision-making, execution) of an individual, this section discusses dynamic evolution of those modules over time (i.e. learning and forgetting). By learning and forgetting, our intent is to represent dynamic accumulation and degradation of experiences or knowledge of an individual represented by the extended BDI framework (and underlying modules), respectively. The first efforts of the authors' group in learning are discussed in (Lee and Son 2009), where a novice and an expert is differentiated by their performance in predicting the environment depending on their familiarity with the area during the planning stage; in other words, the $\mathrm{CI}$ of the expert is higher than that of the novice. To this end, output of the BBN for an expert is further adjusted based on the current value of CI such a way to maximize the CI, where pairs of states (value of CI in our work) and actions (adjustment of output from BBN in our work) are suggested by a reinforcement learning technique (Qlearning algorithm in particular).

Later, the authors' group has continued efforts to formalize both the structural learning as well as parametric learning involving the BBN of the extended BDI, where the structural learning focuses on the network structure of BBN and the parametric learning focuses on the prior distributions between the connected variables in the BBN (Kim and Son 2013). In our earlier work on modeling driver's route choice planning behavior (Kim et al. 2012), three types of drivers were considered: 1) commuters who have full information about the environment; 2) explorers who have partial information about the environment; and 3 ) travelers who have no information of the environment. Figure 3 depicts exemplary BBNs for each of the commuter and explorer, where commuters consider all three environmental variables in evaluating each path available in the intersection along the route, however explorers do not take into account traffic flow rate as an environment variable. We have been developing algorithms to formally represent the dynamic evolution of the BBN of the explorer to that of the commuter based on the Bayesian Structural Expectation Maximization (BSEM) algorithm proposed by Friedman (1998), and our preliminary works are available in Kim and Son (2013).

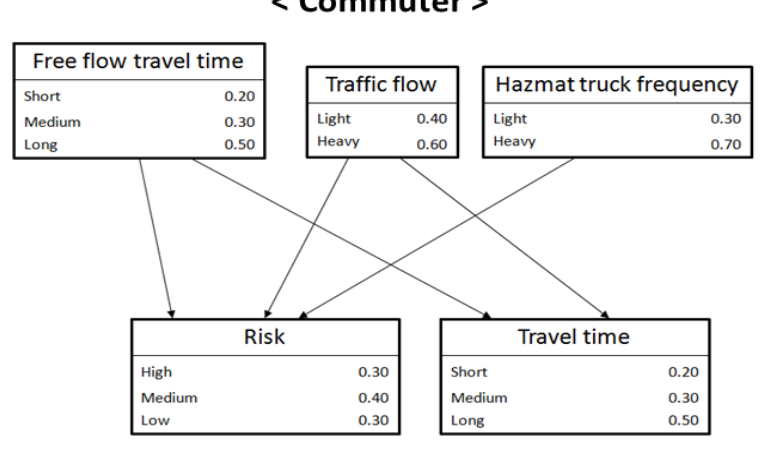

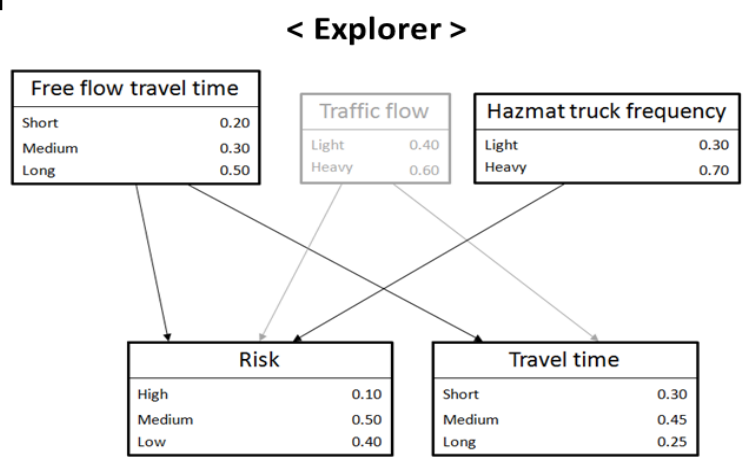

Figure 3: BBNs for a commuter (a) and an explorer (b) in their driving route choice planning behavior considering three conditions of each route (Kim et al. 2012)

\subsection{Human Interactions using the Extended BDI Framework}

This section discusses how human interactions (e.g. competition, collaboration, compromise, accommodation, avoidance) are addressed under the extended BDI framework. In our earlier work (Kim et al. 2012), five major human interactions defined by a social science literature (Thomas 1976) have been successfully addressed for the driver's route choice behavior. Those five interactions are avoidance, accommodation, compromise, collaboration and competition. In our work (Kim et al. 2012), these five interactions are further categorized into two groups, where collaboration, comprise and accommodation 
interactions are triggered by need (the first category), and competition and avoidance interactions are triggered by situation (the second category). Figure 1(b) depicts how the extended BDI framework can be adapted to represent both types of interactions, where Desires, Real-time Planner, and Decision Executor are replaced by Interaction Desire, Interaction Planner, and Interaction Executor, respectively. Basically, our extended BDI framework can be directly used to address both types of interactions. In our work (Kim et al. 2012), how perception of environmental variables (information) of two interacting individuals are affected by each of the five interactions is discussed. For example, (4) and (5) depict updated perception of an environmental variable (information) by the collaboration interaction, where $f_{k}(t)$ is perceived value of an environment by agent $k$ at time $t$. Figure 4 depicts how the five interaction types affected travelers on their total travel time, where information exchange (collaboration, compromise, and accommodation interactions) helped reduce the average travel time (about 1.13 hours) for the travelers.

$$
\begin{gathered}
f_{1}(t)=\frac{C I_{t, 1} f_{1}(t-1)+C I_{t, 2} f_{2}(t-1)}{C I_{t, 1}+C I_{t, 2}} \\
f_{2}(t)=f_{1}(t)
\end{gathered}
$$

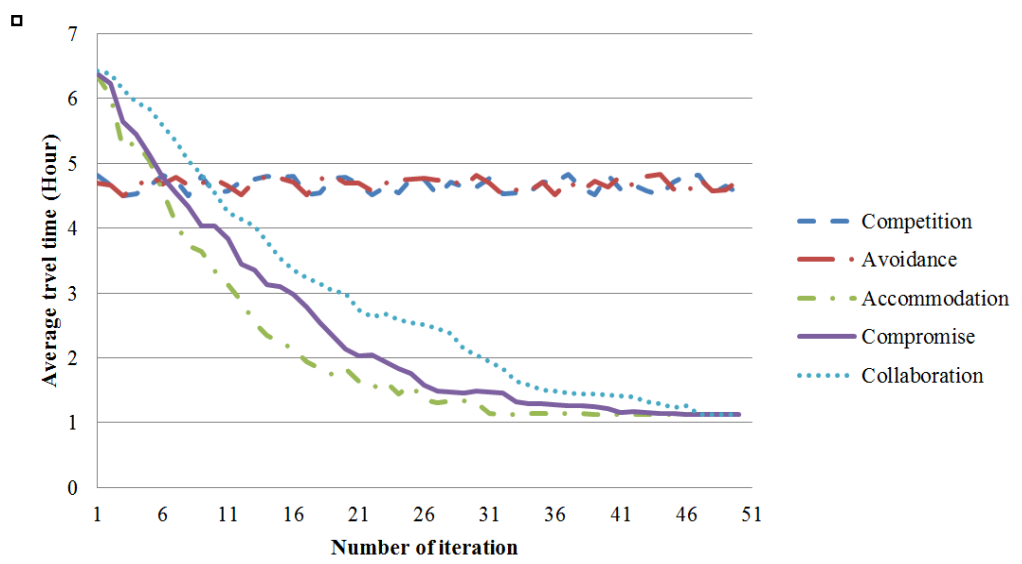

Figure 4: Average travel time of travelers via different interactions (Kim et al. 2012)

\section{VARIOUS APPLICATIONS OF EXTENDED BDI FRAMEWORK}

This section discusses various applications for which the extended BDI framework (see Section 2) has been applied last decade by the authors' group. Figure 5 depicts snapshots of each of the applications, which are briefly described below:

- Emergency evacuation from bomb attack (Lee, Son, and Jin 2010) (see Figure 5(a)): The goal is to model human evacuation behaviors under a bomb attack in the Washington DC mall area, where considered decisions are to select a path at each intersection considering environmental conditions (e.g. intensity of fire, intensity of smoke, existence of policemen, existence of crowd, distance to the destination) of available paths. Two different types of agents (commuters vs. travelers) were developed based on the extended BDI framework depending on the familiarity with the environment. In addition, injury level of individuals are also considered. Human behavior data was collected from human-in-the-loop experiments conducted in a CAVE-based virtual reality environment. The simulation was developed in AnyLogic, where each agent calls Netica (software for BBN) and Soar (implementing probabilistic DFS). The constructed simulation was used 


\section{Son, Kim, Xi, and Mungle}

to test the impact of various factors (e.g. number of police officers) on the average evacuation time and percentage of casualties,

- Complex social network among stakeholders for alliance-based software development (Celik et al. 2011) (see Figure 5(b)): The goal is to develop a simulation model of a social network involving multiple stakeholders in different locations. In particular, a software enhancement request process in Kuali, an alliance-based open source software development project involving 12 organizations, was used as a case study. Data on the work relationships among the members were collected through a survey in which respondents were asked to indicate their working relationships (e.g. communication, coordination, or collaboration, or none) with others. The simulation model was developed in AnyLogic, and was used to predict the short-term performance (e.g. productivity) as well as the long-term performance (e.g. workforce training and robustness of the organization) of the organization for different workforce mixes,

- Surveillance and crowd control via UAVs and UGVs (Khaleghi et al. 2013) (see Figure 5(c)): A simulation model (in both 3D environment as well as GIS environment) has been developed in Repast Simphony, whose major components are human crowd, Unmanned Ground Vehicles (UGVs), and Unmanned Aerial Vehicles (UAVs). In the current model, social force model (Helbing and Molnár 1995) is used to represent individual's physical congestion with other humans as well as obstacles (e.g. rocks), and their decision behaviors will be enhanced by the extended BDI framework in the future. In addition to the fast running simulation model, another version of the same simulation is run in real-time, where UAVs in the simulation interact with a real hardware UAV via message exchanges. The simulation has been used to test the effectiveness of the algorithms coordinating UAVs and UGVs in detecting and tracking the crowd in a rural environment (e.g. desert near the US boarder),

- Shopping behaviors in a mall (Xi and Son 2011) (see Figure 5(d)): Human shopping behaviors are concerned, where both individual customers as well as group customers are considered. Each customer is tagged with male, female, or child. In addition, based on their shopping style, customers are further categorized to planned shoppers (people who visit the mall with a specific shopping plan) and unplanned shoppers. The demographic data of Tucson, AZ was used. The simulation model was constructed in AnyLogic, which has been used to test impact of various factors (e.g. group shopping behavior, arrangement of stores) on the average distance among neighboring shoppers, the movement speed of pedestrians, and the profit of the shopping mall.

- Drivers' behaviors under different cases (Kim, Xi, and Son 2013) (see Figure 5(e)): Drivers' behaviors under three cases (i.e. lane changing, following previous car, dilemma zone) that have been widely studied in the field of transportation are concerned. For each of the considered cases, models have been developed in AnyLogic to demonstrate behaviors of conservative, aggressive, and nominal drivers. For the case of dilemma zone, the authors have been collaborating with the Federal Highway Administration and its sub-contractors to collect behavior data from a humanin-the-loop experiment to be conducted using a driving simulator. Once the data is collected, the developed extended BDI model will be calibrated,

- Emergency evacuation from factory fire (Vasudevan and Son 2011) (see Figure 5(f)): The goal is to model human evacuation behaviors under a factory fire, where the considered decisions are to select an exit (destination), and the paths from the current location to the selected exit. Social force model (Helbing and Molnár 1995) was used to represent individual's physical congestion with other humans as well as obstacles (e.g. walls and machines). Human behavior data was collected from human-in-the-loop experiments conducted in a CAVE-based virtual reality environment. The simulation was developed in AnyLogic, where each agent calls MSBNx (for BBN). The constructed simulation was used to evaluate four different layouts of an automotive powertrain (engine and transmission) manufacturing plant (e.g. process layout, product layout, cellular layout, hybrid layout) in terms of both productivity (throughput) as well as evacuation time, 

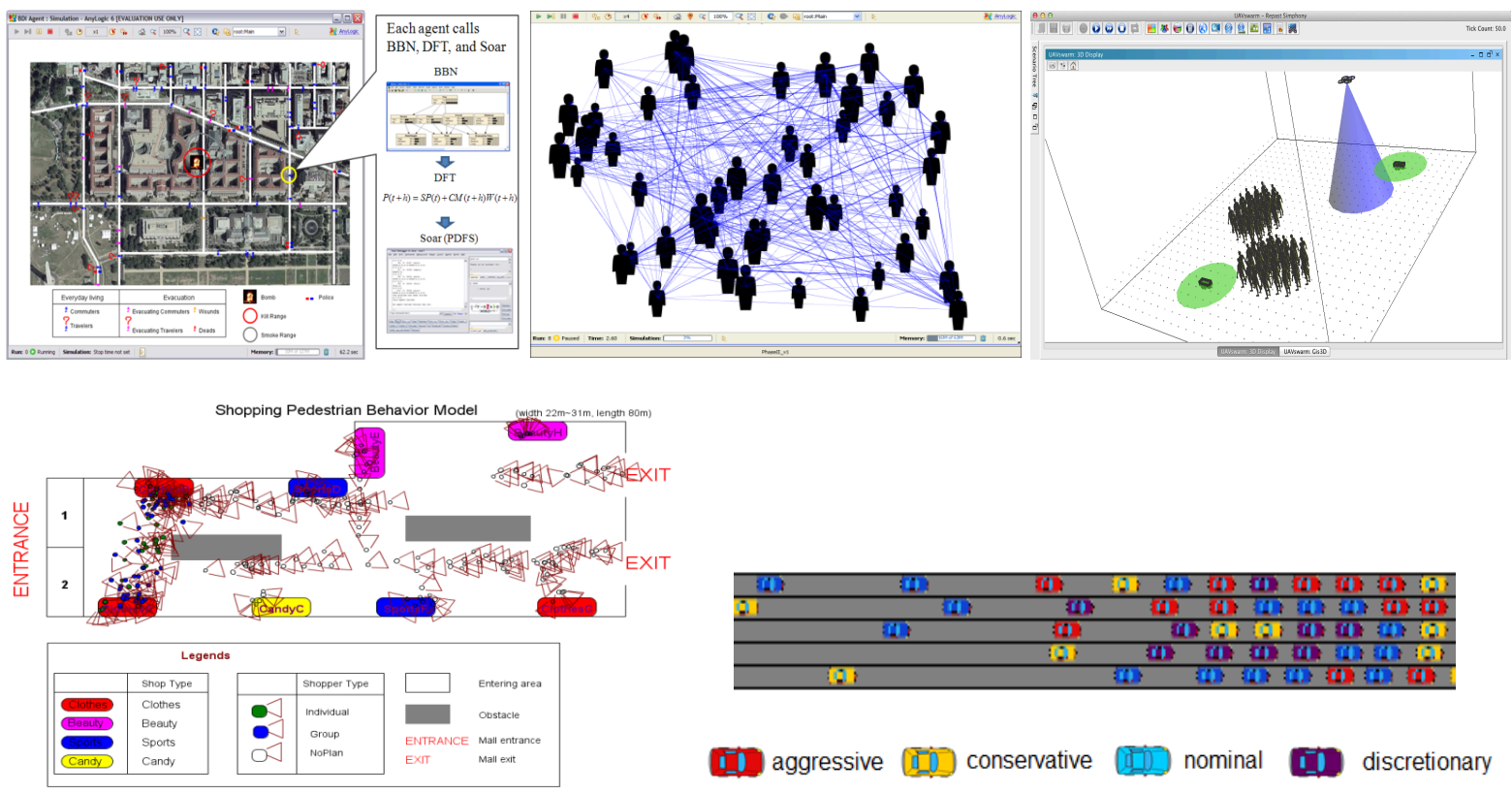

aggressive [0] conservative nominal

I) discretionary
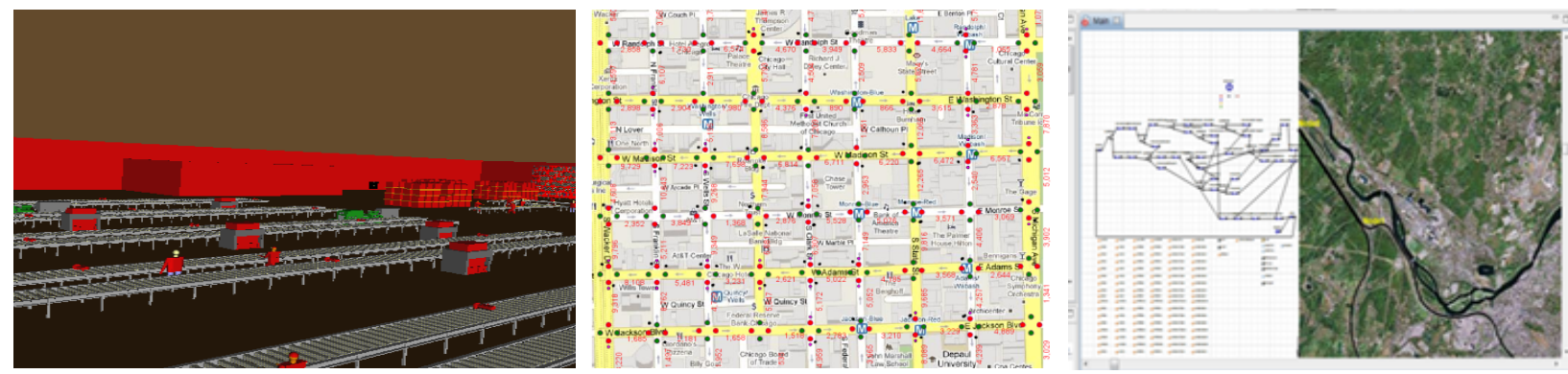

Figure 5: Snapshots of various applications for the extended BDI framework: (a) Emergency evacuation from bomb attack (Lee, Son, and Jin 2010); (b) Complex social network among stakeholders for alliancebased software development (Celik et al. 2011); (c) Surveillance and crowd control via UAVs and UGVs (Khaleghi et al. 2013); (d) Shopping behaviors in a mall (Xi and Son 2011); (e) Drivers' behaviors under different cases (Kim, Xi, and Son 2013); (f) Emergency evacuation from factory fire (Vasudevan and Son 2011); (g) Pedestrian route choice and walking behaviors (Xi and Son 2012); (h) Driver's route choice behaviors considering risk and toll (Kim, Mungle, and Son 2013)

- Pedestrian route choice and walking behaviors (Xi and Son 2012) (see Figure 5(g)): The goal is to model pedestrian's route choice and crossing behaviors at a crosswalk. The DFT (part of the extended BDI framework) is used to represent the psychological preferences of pedestrians for different route choice options. In addition, a Cellular Automata model is used to represent physical interactions among pedestrians and congestions. The simulation model is constructed in AnyLogic based on the real data collected in the Chicago Loop area, and it has been used to test the impact of pedestrian types and traffic signal phase lengths on the average pedestrian waiting time at the crosswalk,

- Driver's route choice behaviors considering risk and toll (Kim, Mungle, and Son 2013) (see Figure 5(h)): The goal is to understand and model driver's route choice behaviors, where each road has different conditions (e.g. frequency of track carrying hazardous materials (hazmat), toll price, free flow travel time, traffic flow, distance from destination). Real traffic data of Albany, NY was 
used to construct a simulation model in AnyLogic, and it was used to test the impact of dual toll pricing policy (imposing toll on both regular traffic as well as hazmat vehicles).

\section{CONCLUSIONS AND FUTURE RESEARCH WORK}

This tutorial has discussed details of the extended BDI framework that the authors' group has been developing and refining last decade, integrating models and techniques from various disciplines such as engineering, psychology, artificial intelligence, and statistics. The tutorial has also introduced various scenarios for which the extended BDI framework has been applied, such as emergency evacuation (from bomb attack as well as factory fire), drivers' behaviors, pedestrian crosswalk behaviors, cyber social network, and shopping behaviors. In addition to major human cognitive functions, the tutorial has also discussed how the extended BDI framework can be extended to handle 1) learning and forgetting behaviors of human and 2) human interactions.

One of the major ongoing research efforts is to reduce the gap between our extended BDI framework with chemical/electrical data that neuroscientist and neurophysiologists collect from human patients and animals (e.g. rats). Figure 6 depicts an interdisciplinary research team, whose members include systems and industrial engineers (the authors' group), neurophysiology, psychology, and recording technology researchers. The goal of such an interdisciplinary team is to reverse-engineer learning and optimal decisionmaking over time and under uncertainty in the mammalian (human and rat) brains. This systematic understanding will form a basis for developing unified, general-purpose engineering algorithms (replicating brain-like creativity) capable of learning to perform optimizations and predictions, using massively parallel computing hardware, in the face of severe complexity, non-convexity, and nonlinearity.

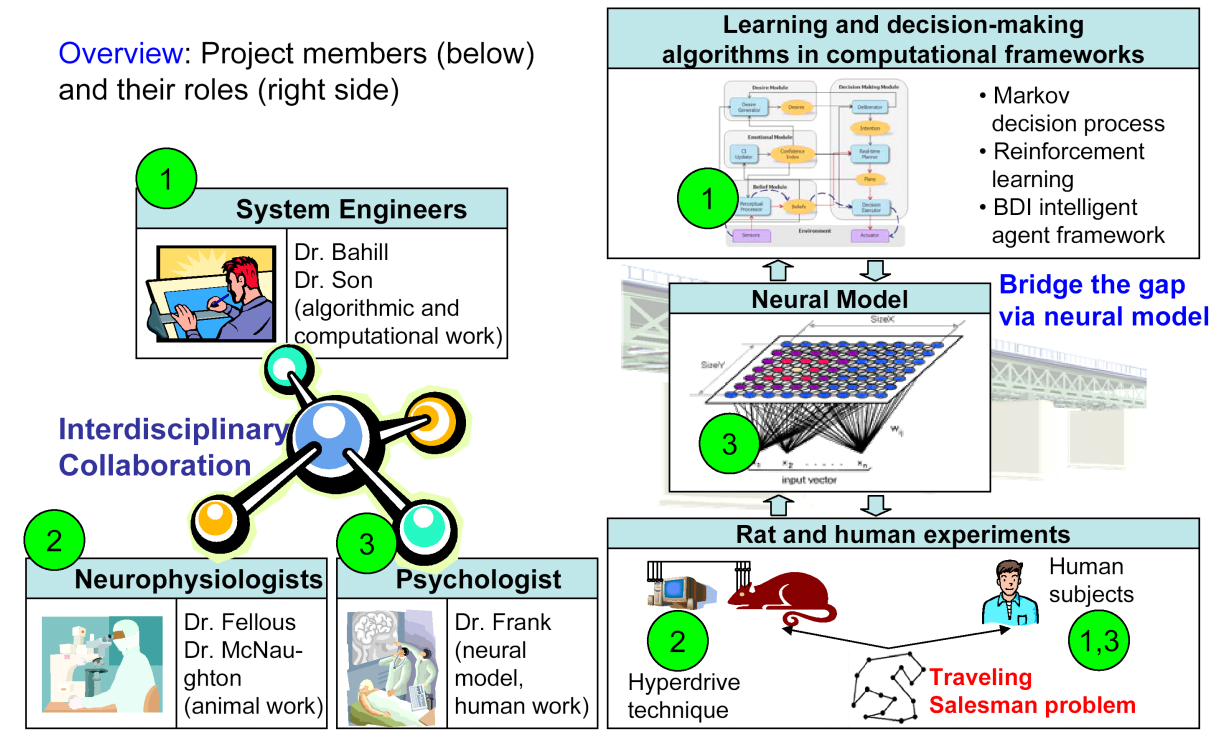

Figure 6: Interdisciplinary research team and overview of future, transformative research

\section{ACKNOWLEDGMENTS}

The research works (methodologies and applications) discussed in this tutorial have been supported by the Air Force Office of Scientific Research under AFOSR/MURI F49620-03-1-0377 and FA9550-12-1-0238, the Arizona Chapter of the Human Factors and Ergonomics Society, the National Science of Foundation under NSF-SOD 0725336 and CMMI-1068866, and Federal Highway Administration under DTFH61-10C-00009 (SA421-0212-6629) and FHWA EAR DTFH61-10-R-00036. 


\section{REFERENCES}

Act-R. 2013. Act-R. Accessed May 25. http://act-r.psy.cmu.edu/.

Bratman, M. 1987. "Intention, Plans, and Practical Reason." CSLI Publications.

Busemeyer, J. and Townsend, J. 1993. "Decision field theory: A dynamic-cognitive approach to decision making in an uncertain environment." Psychological Review 100(3):432-459.

Celik, N., Lee, S., Mazhari, E., Son, Y., Lemaire, R., and Provan, K. 2011. "Simulation-based workforce assignment in a multi-organizational social network for alliance-based software development." Simulation Modelling Practice and Theory 19:2169-2188.

Friedman N. 1998 "The Bayesian structural EM algorithm." In Proceedings of the 14th Conference on Uncertainty in Artificial Intelligence. San Francisco, CA: Morgan Kaufmann.

Helbing, D., Molnár, P. 1995. "Social Force Model for Pedestrian Dynamics". Physics Review E 51(5):4282-4286.

Khaleghi, A., Xu, D., Lobos, A., Minaeian, S. Son, Y., and Liu, J. 2013. "Agent-based Hardware-in-theloop Simulation for Mission Planning and Crowd Control via UAVs and UGVs." In Proceedings of the 2013 Winter Simulation Conference, Edited by R. Pasupathy, S.-H. Kim, A. Tolk, R. Hill, and M. E. Kuhl. Piscataway, New Jersey: Institute of Electrical and Electronics Engineers, Inc.

Kieras, D. E., Meyer, D. E. 1997. "An overview of the EPIC architecture for cognition and performance with application to human-computer interaction." Human-computer interaction 4(12):391-438.

Kim, S., Mungle, S., and Son, Y. 2013. "An Agent-based Simulation Approach for Dual Toll Pricing of Hazardous Material Transportation." In Proceedings of the 2013 Winter Simulation Conference, Edited by R. Pasupathy, S.-H. Kim, A. Tolk, R. Hill, and M. E. Kuhl. Piscataway, New Jersey: Institute of Electrical and Electronics Engineers, Inc.

Kim, S., Son, Y. 2013. "On-line Learning of Bayesian Belief Network for the Evolved Transportation System" Unpublished Technical Report, Department of Systems and Industrial Engineering, The University of Arizona, Tucson, Arizona.

Kim, S., Xi, H., Mungle, S., Son, Y. 2012, "Modeling Human Interactions with Learning under the Extended Belief-Desire-Intention Framework." In Proceedings of the 2012 Industrial and Systems Engineering Research Conference.

Kim, S., Xi, H., Son, Y. 2013. "Scalability of Modeling Driver's Behavior under the Extended BeliefDesire-Intention Framework" Unpublished Technical Report, Department of Systems and Industrial Engineering, The University of Arizona, Tucson, Arizona.

Lee, S., Son, Y. 2009, "Dynamic Learning in Human Decision Behavior under the Extended BeliefDesire-Intention Framework". In Proceedings of the 2009 Industrial Engineering Research Conference.

Lee, S., Son, Y., and Jin, J. 2010. "Integrated human decision making and planning model under extended belief-desire-intention framework." ACM Transactions on Modeling and Computer Simulation (TOMACS), 20(4): 23(1)-23(24).

MIT CogNet. 2013. The Brain Sciences Connection MIT CogNet. Accessed May 25. http://cognet.mit.edu/.

NSF Proposal Solicitation. 2008. "Emerging Frontiers in Research and Innovation 2008 (EFRI-2008): Cognitive Optimization and Prediction: From Neural Systems to Neurotechnology (COPN)."

Soar. 2013. The University of Michigan Soar. Accessed May 25. http://sitemaker.umich.edu/soar/home.

Son, Y. and Jin, J. 2006. "Extended BDI framework and technologies for modelling partial human decision-making," in AFOSR Cognition and Decision Program Review Workshop.

Thomas, K. 1976. "Conflict and conflict management," in Handbook of Industrial and Organizational Psychology, Dunnette, M.D. (eds), Chicago: Rand McNally, Inc., 889-935. 
Vasudevan, K., Son, Y. 2011. "Concurrent Consideration of Evacuation Safety and Productivity in Manufacturing Facility Planning using Multi-Paradigm Simulations." Computers and Industrial Engineering 61:1135-1148.

Wikipedia. 2013. Belief-Desire-Intention (BDI) Software Model. Accessed May 25. https://en.wikipedia.org/wiki/Belief\%E2\%80\%93desire\%E2\%80\%93intention_software_model.

Xi, H., Son, Y. 2012. "Two-Level Modeling Framework for Pedestrian Route Choice and Walking Behaviors." Simulation Modelling Practice and Theory 22:28-46.

Xi, H., Lee, S., Son, Y. 2011. "Chapter 4: An Integrated Pedestrian Behavior Model Based on Extended Decision Field Theory and Social Force Model." In: Rothrock, L., Narayanan, S. (eds.) Human-inthe-loop Simulation: Methods and Practice. Springer.

Zhao, X., and Son, Y. 2008. "BDI-based human decision-making model in automated manufacturing systems." International Journal of Modeling and Simulation 28(3):347-356.

\section{AUTHOR BIOGRAPHIES}

YOUNG-JUN SON is a Professor of Systems and Industrial Engineering at the University of Arizona and Director of Advanced Integration of Manufacturing Systems and Technologies Center. His research focuses on the coordination of a multi-scale, networked-federated simulation and decision model needed for design and control in manufacturing enterprise, renewable energy network, homeland security, agricultural supply network, and social network. He has received several research awards such as the SME 2004 Outstanding Young Manufacturing Engineer Award, the IIE 2005 Outstanding Young Industrial Engineer Award, the IERC Conference Best Paper Awards (2005, 2008, 2009), and Best Paper of the Year Award in 2007 from IJIE. His e-mail and web addresses are son@sie.arizona.edu and http://tucson.sie.arizona.edu/faculty/son/, respectively.

SOJUNG KIM is a Research Assistant in the Department of Systems and Industrial Engineering at the University of Arizona. He received his Master in Industrial and Systems Engineering from Dongguk University, Republic of KOREA in 2010. His research interests include Agent based modeling \& simulation, Human decision making and Behavior modeling. He is a member of INFORMS, and IIE. His email address is sojungkim@email.arizona.edu and his web page is https://sites.google.com/site/sojungk8/.

HUI XI is a Ph.D. student in the Department of Systems and Industrial Engineering at the University of Arizona. She received her BS degree in Automation Measurement and Control from Harbin Institute of Technology in China, and her MS degree in Systems and Industrial Engineering at The University of Arizona in 2010. Her research interests include agent-based simulation and modeling pedestrian (with interactions with vehicles). She is a student member of INFORMS. She can be reached at huix@email.arizona.edu.

SANTOSH MUNGLE is graduated with his master in Systems Engineering from the University of Arizona. He received his first master in Infrastructure Design and Management from Indian Institute of Technology Kharagpur in 2010. He worked as a Visiting Research Engineer at French National Institute for Research in Computer Science and Control (INRIA). His research interests include Heuristics, Multiobjective Optimization, and Simulation Modeling. He is a member of INFORMS. His email address is santoshmungle@gmail.com and his web page is https://sites.google.com/site/santoshmungle/. 